\title{
Genome-wide transcriptional profiling linked to social class in asthma
}

\author{
E Chen, ${ }^{1}$ G E Miller, ${ }^{1}$ H A Walker, ${ }^{1}$ J M Arevalo, ${ }^{2}$ C Y Sung, ${ }^{3,4}$ S W Cole ${ }^{2,3,4}$
}

\begin{abstract}
- Additional methodological data are published online only at http://thorax.bmj.com/content/ vol64/issue 1

${ }^{1}$ University of British Columbia, Department of Psychology, Vancouver, British Columbia, Canada; ${ }^{2}$ UCLA, Department of Medicine, Division of Hematology-Oncology, Los Angeles, California, USA; ${ }^{3}$ UCLA AIDS Institute, UCLA Molecular Biology Institute, Los Angeles, California, USA; ${ }^{4}$ UCLA Norman Cousins Center, Los Angeles, California, USA
\end{abstract}

Correspondence to: Dr E Chen, University of British Columbia, Department of Psychology, 2136 West Mall, Vancouver BC V6T1Z4, Canada; echen@psych.ubc.ca

Received 20 December 2007 Accepted 4 August 2008

\section{ABSTRACT}

Objectives: Low socioeconomic status (SES) is one of the most robust social factors associated with disease morbidity, including more severe asthma in childhood. However, our understanding of the biological processes that explain this link is limited. This study tested whether the social environment could get "under the skin" to alter genomic activity in children with asthma.

Design and participants: Two group design of children with physician diagnosed asthma who came from low or high SES families.

Outcomes: Genome-wide transcriptional profiles from T lymphocytes of children with asthma.

Results: Children with asthma from a low SES background showed overexpression of genes regulating inflammatory processes, including those involved in chemokine activity, stress responses and wound responses, compared with children with asthma from a high SES background. Bioinformatic analysis suggested that decreased activity of cyclic AMP response element binding protein and nuclear factor $\mathrm{Y}$ and increased nuclear factor $\kappa B$ transcriptional signalling mediated these effects. These pathways are known to regulate catecholamine and inflammatory signalling in immune cells.

Conclusions: This study provides the first evidence in a sample of paediatric patients diagnosed with asthma that the larger social environment can affect processes at the genomic level. Specifically, gene transcription control pathways that regulate inflammation and catecholamine signalling were found to vary by SES in children with asthma. Because these pathways are the primary targets of many asthma medications, these findings suggest that the larger social environment may alter molecular mechanisms that have implications for the efficacy of asthma therapeutics.

The larger social environment has been found to affect physical health in individuals across the lifespan. Across all social factors, low socioeconomic status (SES) exhibits one of the strongest and most consistent associations with morbidity and mortality across a wide range of diseases, ${ }^{1}$ including childhood asthma. In particular, children with asthma from a low SES background suffer from more frequent hospitalisations, emergency department visits and more functional impairment (eg, activity limitations, days spent in bed) compared with children from a higher SES background. ${ }^{2-8}$

In seeking to understand how the larger social environment "gets in the body" to impact on the pathophysiology of asthma, researchers have investigated relationships between the social environment and inflammation. In healthy adults, low SES has been linked to heightened levels of circulating inflammatory markers, such as C reactive protein and interleukin (IL) $6 .{ }^{910}$ In children with asthma, low SES has been associated with greater eosinophil counts as well as heightened in vitro stimulated production of inflammatory cytokines implicated in asthma, such as IL5 and IL13. ${ }^{11}{ }^{12}$ Another social factor associated with low SES-stress-has been linked to heightened inflammatory responses to allergen challenge in patients with asthma, ${ }^{13}$ and decreased expression of genes coding hormonal receptors that regulate inflammation, including the glucocorticoid and $\beta$ adrenergic receptors. ${ }^{14}$

Although relationships between SES and inflammation are increasingly recognised, the molecular mechanisms underlying this relationship remain unclear. The present study utilised an in vivo genomics based approach to identify differences in $\mathrm{T}$ cell gene expression associated with SES in a sample of children with asthma, and to identify candidate signal transduction pathways that may shape those differences. Because the focus was on understanding disparities associated with disease progression, we targeted a sample of children with pre-existing asthma.

Based on previous research linking SES and inflammation, we hypothesised that low SES would be associated with alterations in proinflammatory transcription control pathways. Based on previous data implicating the $\beta$ adrenergic and glucorticoid pathways in the relationship between social factors and inflammation, ${ }^{14}$ we hypothesised that transcriptional mediators of these types of hormonal signals would differentiate $\mathrm{T}$ cell gene expression profiles in children from low versus high SES backgrounds. As a second aim, we tested whether social factors related to SES, such as cognitive interpretations, could explain associations between SES and genome-wide transcriptional profiles. Our hypothesis was that SES colours the way in which children interpret their social world, and that these interpretations in turn alter neuroendocrine and inflammatory signalling processes indicated by genome-wide transcriptional profiles.

\section{METHODS}

Additional details are available in the supplement (online only).

\section{Participants}

Participants were recruited from an ongoing longitudinal study of children with asthma aged 918 years. A subset of 16 children from low SES and 15 from high SES backgrounds provided blood samples for this study. The low and high SES 
groups were identified based on those who fell into the bottom and top $15 \%$ on parent education and family income variables.

\section{Measures}

\section{Background characteristics}

Family SES was measured by parent report of annual family income and years of parental education. Medical information included use of asthma medications and asthma severity.

\section{Interpretations of stress}

The Cognitive Appraisal and Understanding of Social Events (CAUSE) videos depict age appropriate ambiguous life situations, such as being asked to talk with a teacher after class. ${ }^{15}$ Children's interpretations are rated by judges, with higher numbers indicating greater perceived threat.

\section{Gene expression arrays}

CD2+ cells were isolated from mononuclear cells by immunomagnetic positive selection (Miltenyi Biotec; Auburn, California, USA), and total RNA was extracted (RNAlater/ RNeasy; Qiagen, Valencia, California, USA). Genome-wide transcription profiling was conducted, as previously described. ${ }^{16-18}$ Differentially expressed genes were identified as those showing $\geqslant 30 \%$ difference in mean expression levels in children from low SES versus high SES backgrounds (false discovery rate of 15\%). ${ }^{16}$ Functional commonalities among differentially expressed genes were identified using the GOstat bioinformatics tool (http://gostat.wehi.edu.au). ${ }^{19}$

\section{Transcriptional mediation analyses}

We used a two sample variant of the Transcription Element Listening System (TELiS) (http://www.telis.ucla.edu) ${ }^{17}$ to analyse differential gene expression data in terms of the prevalence of transcription factor binding motifs (TFBMs) within the promoters of differentially expressed genes, as previously described. ${ }^{20}$

Ancillary analyses used a multivariate generalisation of the analysis of covariance (ANCOVA) ${ }^{21}$ to control for potential confounders which could explain associations between SES and gene expression profiles. Covariates included demographic variables (age, gender), medical variables (asthma severity, use of inhaled corticosteroid medication, use of $\beta$ agonist medication) and interpretations of stress (CAUSE video responses). To confirm that natural killer cell prevalence did not affect the results, the relative concentration of mRNAs encoding CD16 and CD56 were included as covariates.

Table 1 Demographic characteristics of the sample

\begin{tabular}{|c|c|c|c|c|c|c|}
\hline & \multicolumn{3}{|c|}{ Low SES } & \multicolumn{3}{|c|}{ High SES } \\
\hline & $\%$ & Mean & SD & $\%$ & Mean & SD \\
\hline Age (year) & & 12.8 & 3.5 & & 14.9 & 1.9 \\
\hline Gender (\% boys) & 63 & & & 85 & & \\
\hline \multicolumn{7}{|c|}{ Medications (\% using each type) } \\
\hline Inhaled corticosteroid & 75 & & & 80 & & \\
\hline$\beta$ agonist & 75 & & & 87 & & \\
\hline Atopic asthma (\%) & 75 & & & 93 & & \\
\hline Asthma severity (1-4 scale) & & 2.8 & 1.0 & & 2.5 & 0.5 \\
\hline $\mathrm{FEV}_{1} \%$ & & 94.9 & 12.6 & & 99.8 & 17.6 \\
\hline
\end{tabular}

The groups did not differ significantly in terms of gender, medications, atopic asthma, asthma severity or $\mathrm{FEV}_{1} \%$ ( $\mathrm{p}$ values $>0.15$ ). Children in the high SES group were older than children in the low SES group $(p=0.047)$.

$\mathrm{FEV}_{1}$, forced expiratory volume in $1 \mathrm{~s}$; SES, socioeconomic status.

\section{RT-PCR confirmation}

Ten transcripts identified as differentially expressed in microarray analyses were independently assayed by quantitative realtime RT-PCR using TaqMan Gene Expression Assays (Applied Biosystems, Foster City, California, USA), as described in the online supplement.

\section{Clinical variables}

Parents reported on children's daytime and night-time symptoms over the previous 2 weeks, and whether their child went to the emergency department because of asthma in the past 6 months.

\section{RESULTS}

Descriptive information about the sample is shown in table 1. The low and high SES groups did not differ in terms of asthma severity, use of inhaled corticosteroids, use of $\beta$ agonists, forced expiratory volume in $1 \mathrm{~s}$ or atopic status (all $\mathrm{p}$ values $>0.15$ ).

\section{Differential gene expression}

Sixty transcripts were differentially expressed between the low and high SES groups, representing 56 distinct named human genes. Thirty-four reflected overexpression in the low SES group whereas 26 reflected overexpression in the high SES group (table 2).

Genes showing overexpression in children with asthma from a low SES background contained themes of the following: immune response and inflammation (eg, S100A8, S100A9, S100A12, gene ontology terms: GO:0006955, GO:0006954, GO:0002376), chemokine activity (eg, PPBP, PF4, GO:0008009, GO:0042379), stress response (eg, SOD2, ANXA5, TYMS, GO:0006950), wound response (eg, ADM, ANXA5, $\mathrm{GO}: 0009611)$ and antigen processing and presentation (eg, HLA-DQA1, HLA-DQB1, GO:0042613, GO:0042611, GO:0002504, GO:0019882) (all p values <0.05). Some overexpressed genes are linked to more than one GO annotation, so the present results should not be considered statistically independent. However, the converging pattern of results indicates relative activation of inflammatory responses in circulating $\mathrm{T}$ lymphocytes from children with asthma from a low SES background.

In contrast, genes that were overexpressed in children with asthma from a high SES background had functional themes related to methylation (eg, FOS, BTG2, GO:0043414, GO:0032259), protein folding (eg, HSPA1A, HSPA6, GO:0006986, GO:0051789), cell differentiation (eg, JUN, GO:0035026), stress response (eg, H2AFX, DNAJB1, GO:0006950) and antigen processing and presentation (eg, HLA-DQA1, HLA-DQA2, GO:0042613, GO:0002504, GO:0042611, GO:0019882) (all p values <0.05). These patterns suggest a relative activation of genes regulating both inflammatory processes and counter-regulatory responses to inflammation.

Confirmatory RT-PCR analyses assayed expression of 10 transcripts identified as differentially expressed in microarray analyses. Consistent with the estimated false discovery rate of $<15 \%$ in the genome-wide differential expression analyses, all tested transcripts showed statistically significant alterations in expression (at $\mathrm{p}<0.05)$ (table 3 ).

\section{Transcription control pathways}

Bioinformatic analyses were used to identify TFBMs within the promoters of differentially expressed genes. Four distinct 
Table 2 Genes showing differential expression in children with asthma from low and high SES backgrounds

\begin{tabular}{|c|c|c|}
\hline Affymetrix probe ID & Gene symbol & Fold-change \\
\hline 205048_s_at & PSPH & 2.49 \\
\hline 202917_s_at & S100A8 & 1.80 \\
\hline 221728 x_at & XIST & 1.65 \\
\hline 220784_s_at & UTS2 & 1.61 \\
\hline 209480_at & HLA-DQB1 & 1.59 \\
\hline 214146_s_at & PPBP & 1.50 \\
\hline 214218_s_at & XIST & 1.49 \\
\hline 209116_x_at & HBB & 1.49 \\
\hline 200782_at & ANXA5 & 1.47 \\
\hline 201743_at & CD14 & 1.45 \\
\hline 211696_x_at & HBB & 1.45 \\
\hline 202912_at & ADM & 1.44 \\
\hline 214414_x_at & HBA2 & 1.44 \\
\hline 202651_at & LPGAT1 & 1.41 \\
\hline 217941_s_at & ERBB2IP & 1.41 \\
\hline 206390_x_at & PF4 & 1.41 \\
\hline 202503_s_at & KIAA0101 & 1.41 \\
\hline 202602_s_at & HTATSF1 & 1.40 \\
\hline 215223_s_at & SOD2 & 1.40 \\
\hline 217022_s_at & IGHA1///IGHA2 & 1.40 \\
\hline 203535_at & S100A9 & 1.39 \\
\hline 202589_at & TYMS & 1.38 \\
\hline 221731_x_at & CSPG2 & 1.37 \\
\hline 213831_at & HLA-DQA1 & 1.37 \\
\hline 213376_at & ZBTB1 & 1.37 \\
\hline 205863_at & S100A12 & 1.36 \\
\hline 209773_s_at & RRM2 & 1.32 \\
\hline 204192_at & CD37 & 1.32 \\
\hline 217811_at & SELT & 1.31 \\
\hline 217414_x_at & $\mathrm{HBA} 1 / / / \mathrm{HBA} 2$ & 1.31 \\
\hline 212406_s_at & PCMTD2 & 1.31 \\
\hline 217232_x_at & HBB & 1.31 \\
\hline 220330_s_at & SAMSN1 & 1.30 \\
\hline 211745_x_at & HBA1 & 1.30 \\
\hline 201464_x_at & JUN & 0.78 \\
\hline 201236_s_at & BTG2 & 0.77 \\
\hline 221833_at & LONPL & 0.77 \\
\hline 205436_s_at & H2AFX & 0.77 \\
\hline 214469_at & HIST1H2AE & 0.77 \\
\hline 205376_at & INPP4B & 0.77 \\
\hline 214131_at & CYorf15B & 0.77 \\
\hline 200664_s_at & DNAJB1 & 0.77 \\
\hline 214805_at & EIF4A1 & 0.77 \\
\hline 206385_s_at & ANK3 & 0.76 \\
\hline 201465_s_at & JUN & 0.75 \\
\hline 206700_s_at & SMCY & 0.72 \\
\hline 200799_at & HSPA1A & 0.72 \\
\hline 204141_at & TUBB2A & 0.69 \\
\hline 201693_s_at & EGR1 & 0.69 \\
\hline 201694_s_at & EGR1 & 0.68 \\
\hline 200800_s_at & HSPA1A///HSPA1B & 0.65 \\
\hline 209189_at & FOS & 0.65 \\
\hline 219629_at & FAM118A & 0.64 \\
\hline 213418_at & HSPA6 & 0.63 \\
\hline 202768_at & FOSB & 0.60 \\
\hline 212671_s_at & $\begin{array}{l}\text { HLA-DQA1///HLA- } \\
\text { DQA2///LOC650946 }\end{array}$ & 0.60 \\
\hline 205000_at & DDX3Y & 0.55 \\
\hline 202581_at & HSPA1B & 0.50 \\
\hline 201909_at & RPS4Y1 & 0.48 \\
\hline 209728 at & HLA-DRB4 & 0.46 \\
\hline
\end{tabular}

SES, socioeconomic status.
Table 3 RT-PCR confirmation of differential gene expression

\begin{tabular}{llll}
\hline Gene & Microarray ratio* & RT-PCR ratio* & $\begin{array}{l}\text { RT-PCR } \\
\text { p value }\end{array}$ \\
\hline EGR1 & 0.68 & 0.52 & 0.0001 \\
FOS & 0.65 & 0.59 & 0.0001 \\
FOSB & 0.60 & 0.79 & 0.0487 \\
JUN & 0.75 & 0.51 & 0.0001 \\
ADM & 1.44 & 1.52 & 0.0114 \\
SOD2 & 1.40 & 1.25 & 0.0006 \\
S100A8 & 1.80 & 1.65 & 0.0001 \\
S100A9 & 1.39 & 1.74 & 0.0001 \\
S100A12 & 1.36 & 2.30 & 0.0001 \\
XIST & 1.65 & 5.58 & 0.0001
\end{tabular}

*Low SES/high SES.

tp Value from linear model analysis.

RT-PCR, reverse transcription-polymerase chain reaction; SES, socioeconomic status.

hormonal or cytokine signalling pathways were consistently linked to low SES (fig 1). Cyclic AMP response element binding protein (CREB) TFBMs were significantly less prevalent in promoters of genes overexpressed in children with asthma in the low SES group (average $55 \%$ decrease, $p=0.031$ ) (table 4). CREB proteins are key mediators of immune cell transcriptional responses to catecholamines. ${ }^{22}$

A second finding involved decreased prevalence of NF-Y TFBMs in promoters of genes overexpressed in children from a low SES background (average 72\% decrease in promoter TFBM prevalence, $p=0.022$ ). NF-Y is activated by the same cAMP/ PKA signalling pathway as CREB, providing a convergent indication of pathway activation.

Decreased activity of AP1 (Fos/Jun) transcription factors in T cells from children from a low SES background was also found (AP1 $\mathrm{p}=0.046$; VJUN $\mathrm{p}=0.048$ ). Reduced activity of this pathway is consistent with the observed downregulation of the FOS, FOSB and JUN transcripts, which encode key elements of the AP1 transcription factor family.

In addition, a heightened prevalence of NFKB TFBMs in genes overexpressed in the low SES group was found (average 2.54fold increase in promoter TFBM prevalence, $p=0.032$ ). NFKB activates numerous genes involved with inflammation, wound healing and cellular stress responses.

All of the above findings remained significant in sensitivity analyses parametrically varying promoter length and TFBM detection stringency (table 4). Other TFBMs were identified in initial analyses as potential contributors to the observed SES related differences in gene expression. However, these TFBMs did not show consistent statistical significance across parametric sensitivity analyses ( $p$ values $>0.05$ ) (table 4 ). To assess the likelihood that the observed differences might arise by chance, we drew 10000 random samples of 60 transcripts from the population of genes assayed by the Affymetrix U133A high density oligonucleotide array, and computed the magnitude of differential prevalence for the 10 TFBMs identified in initial analyses. Results confirmed that the observed differential distributions of CREB, NF-Y, AP1 and NFאB were unlikely to occur in a random sample of genes $(p<0.05)$ (table 4$)$.

\section{Confounders}

After controlling for age and gender, patterns of associations with CREB, NF-Y and NFKB remained statistically significant (all $p$ values $<0.045$ ), with one exception which was the relationship between SES and CREB controlling for age $(p=0.068)$. Associations also remained significant after 
Figure 1 Transcription factor binding motifs that were differentially represented in children with asthma from a low socioeconomic status (SES) versus a high SES background. Data represent mean (SEM) prevalence of response elements within promoters from each group. AP1, activator protein 1; CREB, cyclic AMP response element binding protein; $N F \kappa B$, nuclear factor $\kappa B$; NF-Y, nuclear factor $Y$. in the promoters of genes overexpressed



controlling for asthma severity, inhaled corticosteroid use and $\beta$ agonist use (all $\mathrm{p}$ values $\leqslant .05$ ). Unlike the other transcription control pathways, indications of association between SES and AP1 activity were rendered non-significant by control for several confounders, including age $(p=0.52)$, gender $(p=0.15)$ and $\beta$ agonist use $(p=0.11)$.

Controlling for cognitive interpretations reduced the relationship between SES and CREB activity to non-significant $(p=0.25)$. The same was true for the relationship between SES and NFKB $(p=0.12)$ and to a lesser extent SES and NF-Y $(p=0.077)$. These analyses suggest that children from the low SES group were more likely to perceive threat in ambiguous situations, and this tendency may activate neuroendocrine processes (impacting on CREB and NF-Y through the cAMP/ PKA pathway) that ultimately impact on inflammatory signalling pathways (eg, NFкB).

Finally, controlling for the prevalence of mRNAs encoding CD16 and CD56 did not significantly alter the results for CREB, $\mathrm{NF}-\mathrm{Y}$ or $\mathrm{NF \kappa B}$ (all $\mathrm{p}$ values $<0.05$ ), suggesting that results are not simply a function of different relative proportions of $\mathrm{T}$ cell versus NK cell subtypes in each group.

\section{Clinical outcomes}

The low and high SES groups also differed in clinical profiles, such that children in the low SES group had greater daytime

Table 4 Bioinformatic indications of transcription factor activity

\begin{tabular}{lllll}
\hline & & $\mathbf{p}$ Value \\
\cline { 3 - 5 } TFBM & Ratio* $^{*}$ & Univariate $\uparrow$ & Random: & Sensitivity \\
\hline V\$CP2_01 & 2.33 & 0.0156 & 0.0114 & 0.0943 \\
V\$TAL1BETAITF2_01 & 7.62 & 0.0166 & 0.0084 & 0.0922 \\
V\$NFY_C & 0.28 & 0.0223 & 0.0212 & 0.0485 \\
V\$CREB_04 & 0.45 & 0.0306 & 0.0334 & 0.0056 \\
V\$NFKB_06 & 2.54 & 0.0316 & 0.0232 & 0.0200 \\
V\$SREBP1_01 & 4.50 & 0.0391 & 0.0316 & 0.0759 \\
V\$COUP_01 & 4.85 & 0.0434 & 0.0360 & 0.0926 \\
V\$HSF2_01 & 0.71 & 0.0435 & 0.0460 & 0.0513 \\
V\$AP1FJ_02 & 0.65 & 0.0464 & 0.0484 & 0.0005 \\
V\$VJUN_01 & 0.20 & 0.0477 & 0.0512 & 0.0342 \\
\hline
\end{tabular}

*TFBM prevalence in promoters of genes overexpressed in T lymphocytes from the low versus the high SES groups.

$\dagger p$ Value from unequal variance Welch t test of TFBM prevalence.

$\$ p$ Value based on 10000 random samples of 60 human genes.

$\S p$ Value for average over nine combinations of three promoter lengths $(-300 \mathrm{bp}$,

$-600 \mathrm{bp},-1000$ to $+200 \mathrm{bp}$ ) and 3 TFBM detection stringencies (MatSim 0.80,

0.90 , and 0.95 )

SES, socioeconomic status; TFBM, transcription factor binding motif.

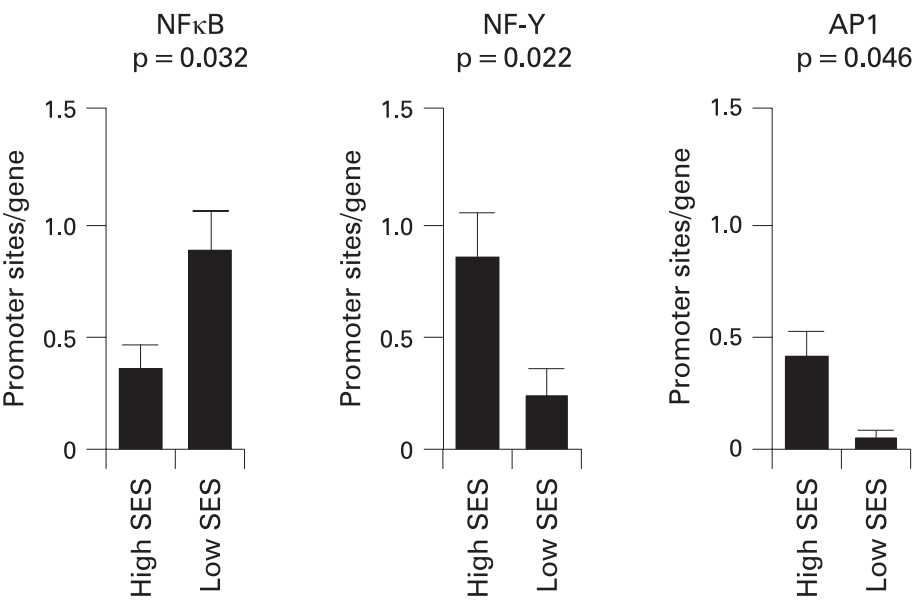

symptoms (low SES mean 5.68 (SD 5.24); high SES mean 1.50 $(\mathrm{SD} 2.10) ; \mathrm{t}=2.77, \mathrm{p}=0.010)$, greater night-time symptoms (low SES mean 2.78 (SD 4.19); high SES mean 0.36 (SD 1.08); $\mathrm{t}=2.10, \mathrm{p}=0.046)$ and showed a trend towards greater emergency department utilisation in the past 6 months because of their asthma (low SES 20\%; high SES $0 \% ; \chi^{2}=3.33$, $\mathrm{p}=0.068)$.

\section{DISCUSSION}

The present study provides the first empirical evidence linking the larger social environment (SES) to $\mathrm{T}$ cell gene expression in the context of a clinical inflammatory disease. Among children with asthma, those from a low SES background showed overexpression of genes regulating a variety of inflammatory processes, including chemokine activity, stress responses and wound responses. Promoter based bioinformatics analyses identified transcription control pathways that may structure the observed patterns of differential gene expression, including decreased CREB, AP1 and NF-Y, and increased NFкB signalling. Children in the low SES group also reported poorer clinical outcomes, such as greater asthma symptoms. Although the cross sectional nature of this study precludes definitive conclusions about causal direction, the findings are consistent with the hypothesis that the larger social environment can get "under the skin" at the level of genomic transcription control pathways.

Genes overexpressed in children in the low SES group included: chemokine ligands such as CXCL4 and CXCL7 which recruit and activate leucocytes; ${ }^{23}$ those involved in antigen processing and presentation (MHC class II protein complexes), a key feature in asthma inflammatory biology; those related to oxidative stress (SOD2), which recruits and activates immune cells, prolonging inflammation; and those related to calcium binding proteins (S100A8, S100A9, S100A12), which have chemotactic effects on leucocytes and proinflammatory effects on endothelial cells. ${ }^{24} 25$ These patterns suggest molecular regulatory mechanisms that may heighten inflammation and worsen clinical outcomes in children with asthma from a low SES background.

Genes overexpressed in children in the high SES group included: those encoding heat shock proteins (HSPA1A, HSPA1B, HSPA6, DNAJB1), which protect cells from inflammatory molecules such as reactive oxygen species, and are protective against pulmonary inflammation ${ }^{26}{ }^{27}$; those involved in cell differentiation and proliferation, such as c-fos and c-jun, which are increased in response to exposure to reactive oxygen 
species $^{28}$; those related to cell cycle control (BTG2), which helps contain the effects of DNA damage ${ }^{29}$; and those involved in the methylation, or silencing, of genes. Note that FOS, FOSB and JUN gene products also encode key components of the AP1 family of transcription factors, which in promoter based bioinformatic analyses showed increased activity in the high SES group. Taken together, these patterns suggest that among children with asthma from a high SES background, heightened inflammation may be counterbalanced by cellular regulatory mechanisms aimed at containing the damage due to inflammation.

This study also identified candidate transcription control pathways that may orchestrate differential patterns of gene expression as a function of SES. Bioinformatic analyses indicated downregulated activity of CREB, AP1 and NF-Y, and upregulated $\mathrm{NF} \kappa \mathrm{B}$ mediated transcription in children with asthma from a low SES background. CREB mediates transcriptional responses to $\beta$ adrenergic receptor signalling through the adenylyl cyclase/cAMP/protein kinase A pathway. ${ }^{22}{ }^{30}$ Adenylyl cyclase activity is impaired after allergen challenge in patients with asthma. ${ }^{31}$ Diminished regulatory signalling from cAMP pathways could lead to increased activation of $\mathrm{T}$ cells, and subsequent expression of Th-2 cytokines. ${ }^{31}$ Reduced CREB signalling may also decrease the efficacy of bronchodilators used as therapeutic agents in asthma. These patterns are consistent with decreased $\beta$ adrenergic receptor mRNA found in lymphocytes of children with asthma with chronic and acute life stress. ${ }^{14}$

Bioinformatic analyses also indicated reduced NF-Y mediated transcription in children with asthma from a low SES background. Like CREB, NF-Y is phosphorylated by PKA, and may thus serve as an indicator of signalling along the $\beta$ adrenergic signalling pathway. ${ }^{32}$ The fact that both transcription factors showed downregulation in the low SES group suggests multiple parallel deficiencies across catecholamine signalling pathways.

Bioinformatic analyses also indicated upregulated $\mathrm{NF} \kappa \mathrm{B}$ signalling in children with asthma in the low SES group. $N F \kappa B$ transactivates a wide variety of inflammatory mediators. Some data suggest that elevated cAMP activity can inhibit $N F \kappa B$ activity ${ }^{33}$; hence increased $N F \kappa B$ and decreased CREB signalling could represent a common regulatory alteration that shifts gene expression profiles towards a more inflammatory phenotype in children from low SES backgrounds.

Our findings are consistent with previous research that has investigated functional immune measures. For example, children with asthma from a low SES background show greater production of Th-2 cytokines and greater eosinophil counts compared with those from a high SES background. ${ }^{12}$ Greater stress has been linked to in vivo inflammatory responses to allergen challenge and in vitro cytokine production in patients with asthma ${ }^{134}$ and predisposed to allergic disease. ${ }^{35}$ Furthermore, stress has been linked to reduced expression of genes coding for hormonal receptors that regulate inflammation. ${ }^{14}$

Statistical analyses revealed that interpretations of stress accounts for some of the SES related differences in indicators of CREB and NFKB activity. This suggests that in order for the social environment to have biological effects, it may have to first be perceived in a threatening manner. In turn, these cognitive perceptions may come with biological costs in transcriptional regulation and inflammatory biology. We note there could be numerous other pathways linking SES to genomic patterns, which should be tested in future studies.
It is unclear whether similar effects would be found in healthy children. However, even if similar effects were evident, these biological mechanisms could still have different implications for those with a pre-existing inflammatory disease. Nonetheless, it would be important for future research to test the effects of SES on gene expression profiles in healthy individuals as well.

Limitations include the observational design, precluding conclusions about causality and directionality. This is a necessary limitation to human work involving social factors that are difficult to randomly assign (eg, socioeconomic status). Secondly, the sample size is small by epidemiological standards, although not unusual for genome-wide microarray studies. One concern about small samples is insufficient power; however, the significant differences in several transcription control pathways suggest that the magnitude of effects is large enough to be detected in a sample of this size. A second concern is whether the findings will be robust and reliable. However, note that the bioinformatics approach used in this study capitalises on the data from thousands of genes to form aggregate and more reliable indicators of transcription factor activity. Thirdly, microarray technology is sometimes criticised as being too exploratory. In this study, however, microarray data were used to test an a priori hypothesis of SES increases in inflammation due to specific hormonal signalling pathways documented in previous research. ${ }^{12}{ }^{14}$ Furthermore, the use of false discovery rate analysis is a standard approach in the genomic literature for adjusting statistics to account for multiple comparisons. Nevertheless, replicating these results in other samples would be important in future studies. Finally, selection of the CD2 antigen to isolate $\mathrm{T}$ lymphocytes may have permitted contamination by natural killer (NK) cells. (CD3 markers were not used because the immunomagnetic separation process for CD3 would have activated $\mathrm{T}$ cells.) However, the present results were not significantly altered in analyses controlling for NK cell marker mRNA.

The present data identified a distinct transcriptional fingerprint of low SES environments, and candidate transcription control pathways structuring those differences, in $\mathrm{T}$ lymphocytes from children with asthma. Children with asthma from a low SES background showed overexpression of genes related to inflammation, chemokine activity, and stress and wound responses, and bioinformatics indications of reduced CREB, AP1 and NF-Y, and increased NFKB signalling. This study provides the first clinical evidence in asthma that broader social environments affect processes at the genomic level, specifically in terms of transcription control pathways that regulate inflammation and catecholamine signalling. Because these pathways are the primary targets of many asthma medications, these findings suggest that the larger social environment may also affect the efficacy of asthma therapeutics. Finally, perceptions of stress play an important role in explaining how SES gets transduced into alterations at the genomic level. Overall, these findings provide new insights into the mechanisms by which social factors affect the course of inflammatory diseases, and highlight the need for future research investigating how the pathophysiology of asthma is shaped by social environments.

Funding: This study was supported by NIH grant HL073975 and the Canadian Institutes of Health Research.

Competing interests: None.

Ethics approval: Ethics approval was obtained. 


\section{REFERENCES}

1. Starfield B, Robertson J, Riley AW. Social class gradients and health in childhood. Ambul Pediatr 2002:2:238-46.

2. Chen $\mathbf{E}$, Matthews KA, Boyce WT. Socioeconomic differences in children's health: How and why do these relationships change with age? Psychol Bull 2002;128:295-329.

3. Maziak W, von Mutius E, Keil U, et al. Predictors of health care utilization of children with asthma in the community. Pediatr Allergy Immunol 2004;15:166-71.

4. Amre DK, Infante-Rivard C, Gautrin D, et al. Socioeconomic status and utilization of health care services among asthmatic children. J Asthma 2002;39:625-31.

5. Dales RE, Choi B, Chen $Y$, et al. Influence of family income on hospital visits for asthma among Canadian school children. Thorax 2002:57:513-17.

6. Simon PA, Zeng ZW, Wold CM, et al. Prevalence of childhood asthma and associated morbidity in Los Angeles County: Impacts of race/ethnicity and income. $J$ Asthma 2003:40:535-43.

7. Miller JE. The effects of race/ethnicity and income on early childhood asthma prevalence and health care use. Am J Public Health 2000;90:428-30.

8. Halfon N, Newacheck PW. Childhood asthma and poverty: Differential impacts and utilization of health services. Pediatrics 1993;91:56-61.

9. Panagiotakos DB, Pitsavos C, Manios Y, et al. Socio-economic status in relation to risk factors associated with cardiovascular disease, in healthy individuals from the ATTICA study. Eur J Cardiovasc Prev Rehabil 2005;12:68-74.

10. Hemingway $\mathbf{H}$, Shipley M, Mullen MJ, et al. Social and psychosocial influences on inflammatory markers and vascular function in civil servants (The Whitehall II study). Am J Cardiol 2003;92:984-7.

11. Chen E, Fisher EB Jr, Bacharier LB, et al. Socioeconomic status, stress, and immune markers in adolescents with asthma. Psychosom Med 2003;65:984-92.

12. Chen E, Hanson MD, Paterson LQ, et al. Socioeconomic status and inflammatory processes in childhood asthma: The role of psychological stress. J Allergy Clin Immunol 2006;117:1014-20.

13. Liu LY, Coe CL, Swenson CA, et al. School examinations enhance airway inflammation to antigen challenge. Am J Respir Crit Care Med 2002;165:1062-7.

14. Miller GE, Chen E. Life stress and diminished expression of genes encoding glucocorticoid receptor and beta(2)-adrenergic receptor in children with asthma. Proc Natl Acad Sci 2006;103:5496-501

15. Chen E, Matthews KA. Development of the Cognitive Appraisal and Understanding of Social Events (CAUSE) videos. Health Psychol 2003;22:106-10.

16. Cole SW, Galic Z, Zack JA. Controlling false negative errors in microarray differential expression analysis: A PRIM approach. Bioinformatics 2003;19:1808-16.

17. Cole SW, Yan W, Galic Z, et al. Expression-based monitoring of transcription factor activity: The TELiS database. Bioinformatics 2005;21:803-10.

18. Bolstad BM, Irizarry RA, Astrand M, et al. A comparison of normalization methods for high density oligonucleotide array data based on bias and variance. Bioinformatics 2003;19:185-93.
19. Beissbarth T, Speed TP. GOstat: Find statistically overrepresented gene ontologies within a group of genes. Bioinformatics 2004;6:1464-5.

20. Wingender $\mathbf{E}$, Dietze $\mathrm{P}$, Karas $\mathrm{H}$, et al. TRANSFAC: A database on transcription factors and their DNA binding sites. Nucleic Acids Res 1996;24:238-41.

21. Miller RG. Beyond ANOVA: Basics of applied statistics. New York, NY: Wiley, 1986

22. Kammer GM. The adenylate cyclase-cAMP-protein kinase A pathway and regulation of the immune response. Immunol Today 1988;9:222-9.

23. Lukacs NW, Miller AL, Hogaboam CM. Chemokine receptors in asthma: Searching for the correct immune targets. J Immunol 2003;171:11-15

24. Lackmann M, Rajasekariah P, Lismaa SE, et al. Identification of a chemotactic domain of the pro-inflammatory S100 protein CP-10. J Immunol 1993:150:2981-91.

25. Hofmann MA, Drury S, Fu CF, et al. RAGE mediates a novel proinflammatory axis: A central cell surface receptor for S100/calgranulin polypeptides. Cell 1999;97:889-901.

26. Jacquiersarlin MR, Fuller K, Dinhxuan AT, et al. Protective effects of HSP70 in inflammation. Experientia 1994;50:1031-8.

27. Villar J, Edelson JD, Post M, et al. Induction of heat stress proteins is associated with decreased mortality in an animal model of acute lung injury. Am Rev Respir Dis 1993:147:177-81.

28. Janssen YMW, Matalon S, Mossman BT. Differential induction of c-fos, c-jun, and apoptosis in lung epithelial cells exposed to ROS or RNS. Am J Physio Lung Cell Mol Physiol 1997;17:L789-96.

29. Rouault JP, Falette N, Guehenneux F, et al. Identification of BTG2, an antiproliferative $\mathrm{p53}$-dependent component of the DNA damage cellular response pathway. Nature Genet 1996;14:482-6.

30. Sanders VM, Straub RH. Norepinephrine, the beta-adrenergic receptor, and immunity. Brain Behav Immunity 2002;16:290-332.

31. Borger P, Postma DS, Vellenga $E$, et al. Regulation of asthma-related T-cell cytokines by the cyclic AMP-dependent signalling pathway. Clin Exp Allergy 2000;30:920-6.

32. Kapatos G, Vunnava P, Wu Y. Protein kinase A-dependent recruitment of RNA polymerase II, C/EBPbeta and NF-Y to the rat GTP cyclohydrolase I proximal promote occurs without alterations in histone acetylation. J Neurochem 2007:101:1119-33.

33. Elenkov IJ, Wilder RL, Chrousos GP, et al. The sympathetic nerve-An integrative interface between two supersystems: The brain and the immune system. Pharmacol Rev 2000;52:595-638.

34. Kang D, Coe C, McCarthy D0, et al. Cytokine profiles of stimulated blood lymphocytes in asthmatic and healthy adolescents across the school year. J Interferon Cytokine Res 1997;17:481-7.

35. Wright RJ, Finn P, Contreras JP, et al. Chronic caregiver stress and IgE expression, allergen-induced proliferation, and cytokine profiles in a birth cohort predisposed to atopy. J Allergy Clin Immunol 2004;113:1051-7. 